\title{
Comparison of the Use of Three Different Stool Preservatives on the Morphology of Intestinal Parasites
}

\author{
P.P. Maneesha, Nonika Rajkumari*, R. Sneha, \\ Shashiraja Padukone and Ajay Philips Selvaratthinam
}

\author{
Department of Microbiology, Jawaharlal Institute of Postgraduate Medical Education and \\ Research, Puducherry-605006, India
}

*Corresponding author

\section{A B S T R A C T}

\begin{tabular}{|l|}
\hline Ke y w o r d s \\
Preservative, \\
Parasite \\
morphology, \\
Formalin, Low \\
viscosity poly vinyl \\
alcohol, Sodium \\
acetate-acetic acid - \\
formalin
\end{tabular}

\section{Keywords}

Preservative, morphology, Formalin, Low alcohol, Sodium acetate-acetic acid -
Stool is one of the most difficult laboratory samples to be handled especially for recovery of intestinal parasites. The preservation of stool parasites faces a lot of difficulties in laboratory and the use of powerful preservatives leads to a better result. This study describes the efficacy of three stool preservatives for the stool parasites and they are $10 \%$ formalin, low viscosity polyvinyl alcohol and sodium acetate acetic acid formalin and compare them using fresh stool which were positive for intestinal parasites. Stool samples positive for an intestinal parasite by microscopy were taken and the outcome was compared after preserving the sample with each of the 3 preservatives and observed after 1 month period in relation to their morphology and staining properties. Of all the 3 preservatives, morphologic identification was more satisfactory with $10 \%$ formalin and least with low viscosity polyvinyl alcohol. However, all didn't give much satisfying results with a permanent stain like trichrome though the best result both in terms of morphological identification and proper taking up of the stain was seen in those preserved with $10 \%$ formalin followed by SAF and least in those with LV-PVA. Using stool preservative is another viable alternative to using fresh stool and it helps to retain the morphology to a certain extent.

\section{Introduction}

Throughout the world, intestinal parasitic infections are endemic and it leads to increased morbidity in many developing countries(1). The main way of transmission can be hand-hand or through the contact with food or water which is contaminated. The main factors which contribute to the prevalence of intestinal parasites are the factors like economic situations, varying climatic conditions, cultural differences and types of sanitation practices. The main age group affected are school going children. The common intestinal parasites are Ascaris lumbricoides, hookworms, Trichuris trichiura, Hymenolepis nana and the protozoan Giardia duodenalis. Recent reports suggests that more than one billion are parasitize worldwide (2).Severe problems are seen in sub-Saharan 
Africa, Asia and Latin America where they are more associated with environmental sanitation, inadequate water supply, fast population growth and other socio-economic problems.

Faeces are the most common specimen collected and examined for demonstration of parasites of the gastrointestinal tract (3). Such specimen are preferably used as freshly collected ones as longer duration and storage can cause the parasites to die or can be overgrown with the normal bacterial flora. Hence, keeping a stool sample for a longer duration needs a form of preservative or a fixative or storage in ultra- low temperatures if it is to be used after a long duration. The widely used preservatives for helminth eggs or protozoan cysts and trophozoites are mercuric chloride and formalin based low- viscosity polyvinyl alcohol (4). Direct wet mount and wet mount prepared from concentration method are performed routinely. The confirmation of the presence of parasite is done by examining the permanent stained smear. Hence, with this aim to see the outcome of the intestinal parasites from stool samples with respect to preservatives, we have conducted this study.

\section{Materials and Methods}

A cross sectional laboratory study was done from January to November, 2018. The study included a total of 25 positive stool samples. All consecutive fresh stool samples of adult or pediatric patients received in the Parasitology laboratory of Microbiology department for routine screening and positive for any intestinal parasite(s) by microscopy during this time period were included in the study after de-identification. Exclusion criteria includes those whose stool samples were less and inadequate and the sample quality was poor irrespective of the positivity. All the samples enrolled for the study were given a random number and were screened for parasites by microscopy.

We explored and analyzed the effect of three different stool preservatives on fecal smears after wet mount and staining for the recovery of parasites. The various preservatives used were $10 \%$ formalin, low viscosity polyvinyl alcohol (LV-PVA) and sodium acetate-acetic acid -formalin (SAF). $10 \%$ formalin, low viscosity PVA and SAF were prepared as per the standard protocol $(5,6)$. Another independent person renumbered the positive samples before putting it into the stool preservative. After putting each positive stool sample in all the 3 preservatives, it was kept for a period of 1 month at room temperature so that the same test were performed and interpreted without any bias afterwards (figure 1). The following effects both pre and post preserved stool samples were observed and noted: (1) effect of preservatives on the morphology of intestinal parasites and (2) comparing the efficacy of the different preservatives.

\section{Pre preservative procedure and microscopy}

The fresh fecal samples were aliquoted into 2 parts. One part was used for pre preservative/fresh procedures and the other part for preservation. Taking the $1^{\text {st }}$ part, it was subdivided into 2 parts again. One part were used for wet mount preparation both by saline and iodine methods along with fecal smear staining using permanent stool staining methods like trichrome and modified acid fast. After this, suitable concentration technique was performed on the second stool sample and the prepared smear using saline and iodine wet mount were subjected to microscopic examination for identification of parasite(s) in the fecal sample and identification were noted (7). Permanent stool staining methods as mentioned above were done to check for quality of smear, recoverability/morphology 
of the parasite and also for diagnosis of the disease (8). Formal ether concentration is the method of choice for routine use by most clinical laboratories (9). Floatation technique like Sheather's sugar solution was seen to be the best concentration technique for coccidian parasites (10).

\section{Preservative procedure}

Only the fresh stool sample shown positive for intestinal parasite(s) by microscopy (direct and concentration methods) were taken for the preservative procedure. Taking the $2^{\text {nd }}$ part of the fresh stool sample, it was aliquoted into 3 different storage vials containing the following preservatives (10\% formalin, low viscosity PVA, SAF). Each vial were filled with $3 \mathrm{ml}$ preservative (vial A-10\% formalin, vial B-low viscosity poly vinyl alcohol, vial 3- sodium acetate-acetic acid-formalin) after $1 \mathrm{mg}$ of the sample (5) was transferred in the container. These fecal samples were preserved in a container at room temperature for 1 month (4).

\section{Post preservative procedure and microscopy}

After 1 month, the samples were taken out and from the preserved sample; a wet mount of direct, concentration and a fecal smear was prepared from each of the vials containing the 3 different preservatives and stained with trichrome stain and modified acid fast stain (7). The microscopic results were noted and compared with the pre-preservative findings.

\section{Interpretation of results}

For the purpose of this study, 2 persons checked the results and were noted down. The morphological identification, the parasites were confirmed by the concerned faculty. The results of either wet or stained smears; both pre and post preservative were characterized as satisfactory or unsatisfactory. Satisfactory if the microscopic picture was of textbook quality, parasitic identification was possible and diagnosis of infection was also possible. Microscopic results were categorized as unsatisfactory if extreme morphologic distortion or barely recognizable structures were seen or diagnosis of infection was difficult or impossible after microscopy (4). These were done for all the wet mounts and for all the stained smears.

\section{Statistical analysis}

The number of samples used for this study was 25. All of the stool samples were positive for either cysts or eggs of intestinal parasite(s). This was calculated by analyzing the number of stool positive for parasites which were received in the Microbiology department. Categorical variables like microscopy positivity, quality of smear were summarized by using frequencies and percentages. Comparison of above variables before and after addition of preservatives were compared using McNemar Chi squared test(paired design)and $\mathrm{P}$ value of less than 0.05 were considered to be statistically significant.

\section{Results and Discussion}

Of the 25 positives, 7 of them were positive for hookworm eggs, 8 were positive for cysts of Giardia lamblia, 3 were positive for Trichuris trichiura eggs, 3 were positive for Ascaris lumbricoides eggs, 3 were positive for Hymenolepis nana eggs, 1 were positive for cyst of Entamoeba histolytical dispar/ moshkovskii/ bangladeshi.

\section{Pre-preservative findings}

In the present study, a total of 25 stool samples positive for an intestinal parasite were taken consecutively (Table no. 1). Of the wet mount, saline has an advantage over iodine to 
detect the motility of trophozoites, whereas iodine had the disadvantage where motility was not appreciated properly. The main advantage of iodine over saline is that the internal structure of parasitic forms can be better seen. Of the 25 parasites, all the $25(100 \%)$ were identified in direct wet mount and only $10(40 \%)$ were identified in direct staining with a permanent stain like trichrome. In case of concentration from the fresh sample, all the 25 (100\%) were recovered but only $5(20 \%)$ were identified after staining from concentration (Figures 2 and 3 ).

\section{Preservative procedure}

The results of positive stool samples preserved in $10 \%$ formalin, low viscosity PVA and SAF. was analyzed after one month after keeping in room temperature and was compared with the pre preservative findings.

\section{Parasite recovery}

The positive samples preserved were analyzed based on the morphology in different preservatives.

\section{Formalin}

While comparing the pre-preservative to the specimens in $10 \%$ formalin, all parasites which were seen in pre-preservative were also recovered in $10 \%$ formalin after 1 month. In this case, $\mathrm{P}$ value was not applicable since the result is $100 \%$ (Table no.2). Direct wet mount from the $10 \%$ formalin preserved ones recovered the parasites only from 22 samples whereas concentration from the same recovered $100 \%(25)$ (Table no 2). The result of smears made from preserved specimen on analysis by permanent staining using trichrome was not satisfactory when compared to the permanent stained smears from direct sample. The morphologic quality and the parasite density were observed to be the main reason for the unsatisfactory results.

\section{LV-PVA}

The comparison between pre preservative and low viscosity PVA showed that, of those 25 which were preserved in low viscosity PVA, only $19(76 \%)$ were recovered after the period of one month. The $\mathrm{P}$ value in this case was 0.005 which showed a significant difference. Direct wet mount of samples preserved in low viscosity PVA were unsatisfactory in terms of proper identification but 19 of them were identified to a discernable level after concentration using formal ether sedimentation method (Table no 2). This maybe due to the visualization of more number of parasites after concentration, in contrast to those without the procedure. The result analyzed after trichrome staining were not satisfactory especially in regards to the morphology though the stain was taken up. The morphologic identification can be done in the 19 samples but lacks the distinctive textbook like quality.

\section{SAF}

It was seen on comparison between prepreservative and after preservation that, of the 25 , only $22(88 \%)$ were recovered after one month in SAF preservative.(Table no.2) The $\mathrm{P}$ value in this case was 0.065 which was not a significant difference. Both types of wet mounts were able to recover the parasites in the 22 samples but it was identifiable in regards to morphology though some amount of morphological distortion was there. The permanent stained smears were not satisfactory for the analysis. There was extreme morphologic distortion or barely recognizable in the remaining 3 samples both in terms of the wet as well as stained smears.

The comparison between the 3 preservatives was shown in the table 3 . All 25 were 
identifiable after one month of preservation in $10 \%$ formalin. While in case of low viscosity PVA, only 19 were identified satisfactorily after one month of preservation and in case of SAF only 22 were identified after one month of preservation. The $\mathrm{P}$ value in this case was 0.03 , which shows significant difference. Of the 25 samples, the parasites were better preserved by $10 \%$ formalin compared to low viscosity PVA and SAF. This is true more so with regards to morphological identification than that of taking up the permanent stains. The recoveries of parasitic forms were much better with the concentration technique compared to the direct wet mount (Figures 4 and 5). The concentration used here was formal ether sedimentation. It was the helminthic eggs which were better preserved than protozoan cyst in all the three preservatives especially in terms with morphology. Permanent staining techniques like trichrome which were also used to analyze the parasitic forms showed the result by staining techniques were not satisfactory to identify the parasitic forms. This was more in terms with LV-PVA and SAF compared to $10 \%$ formalin. The smear which was made directly from afresh positive stool sample gave satisfactory result for the identification of parasitic forms than those made after preservation.

Of all the 3 preservatives, morphologic identification was more satisfactory with $10 \%$ formalin and least with LV-PVA. However, all didn't give much satisfying results with a permanent stain like trichrome though the best result both in terms of morphological identification and proper taking up of the stain was seen in those preserved with 10\% formalin followed by SAF and least in those with LV-PVA.

Table.1 Distribution of intestinal parasites

\begin{tabular}{|l|l|l|}
\hline Parasite & Number & Percentage (\%) \\
\hline Hookworm & 7 & 28 \\
\hline Trichuris trichiura & 3 & 12 \\
\hline Giardia lamblia & 8 & 32 \\
\hline $\begin{array}{l}\text { Entamoeba histolytica/ dispar } \\
\text { /moshkovskii/ bangladeshi }\end{array}$ & 1 & 4 \\
\hline Ascaris lumbricoides & 3 & 12 \\
\hline Hymenolepis nana & 3 & 12 \\
\hline
\end{tabular}

Table.2 Comparison of three preservatives with pre-preservative findings

\begin{tabular}{|c|c|c|c|}
\hline & Number & $\%$ & P value \\
\hline Pre preservative & 25 & 100 & NA \\
\hline 10\% formalin & 25 & 100 & NA \\
\hline $\begin{array}{c}\text { Low viscosity } \\
\text { Polyvinyl alcohol }\end{array}$ & 19 & 76 & $0.005^{*}$ \\
\hline $\begin{array}{c}\text { Sodium acetate } \\
\text { acetic acid formalin }\end{array}$ & 22 & 88 & 0.065 \\
\hline
\end{tabular}


Table.3 Comparison between the findings of the three preservatives

\begin{tabular}{|c|c|c|c|}
\hline & Satisfactory & Unsatisfactory & Percentage (\%) \\
\hline $\begin{array}{c}\text { Low viscosity Poly Vinyl } \\
\text { Alcohol }\end{array}$ & 25 & 0 & 100 \\
\hline $\begin{array}{c}\text { Sodium acetate acetic } \\
\text { acid formalin }\end{array}$ & 19 & 6 & 76 \\
\hline Total = 25 & 22 & 3 & 88 \\
\hline
\end{tabular}

Figure.1 Flowchart of the sample processing

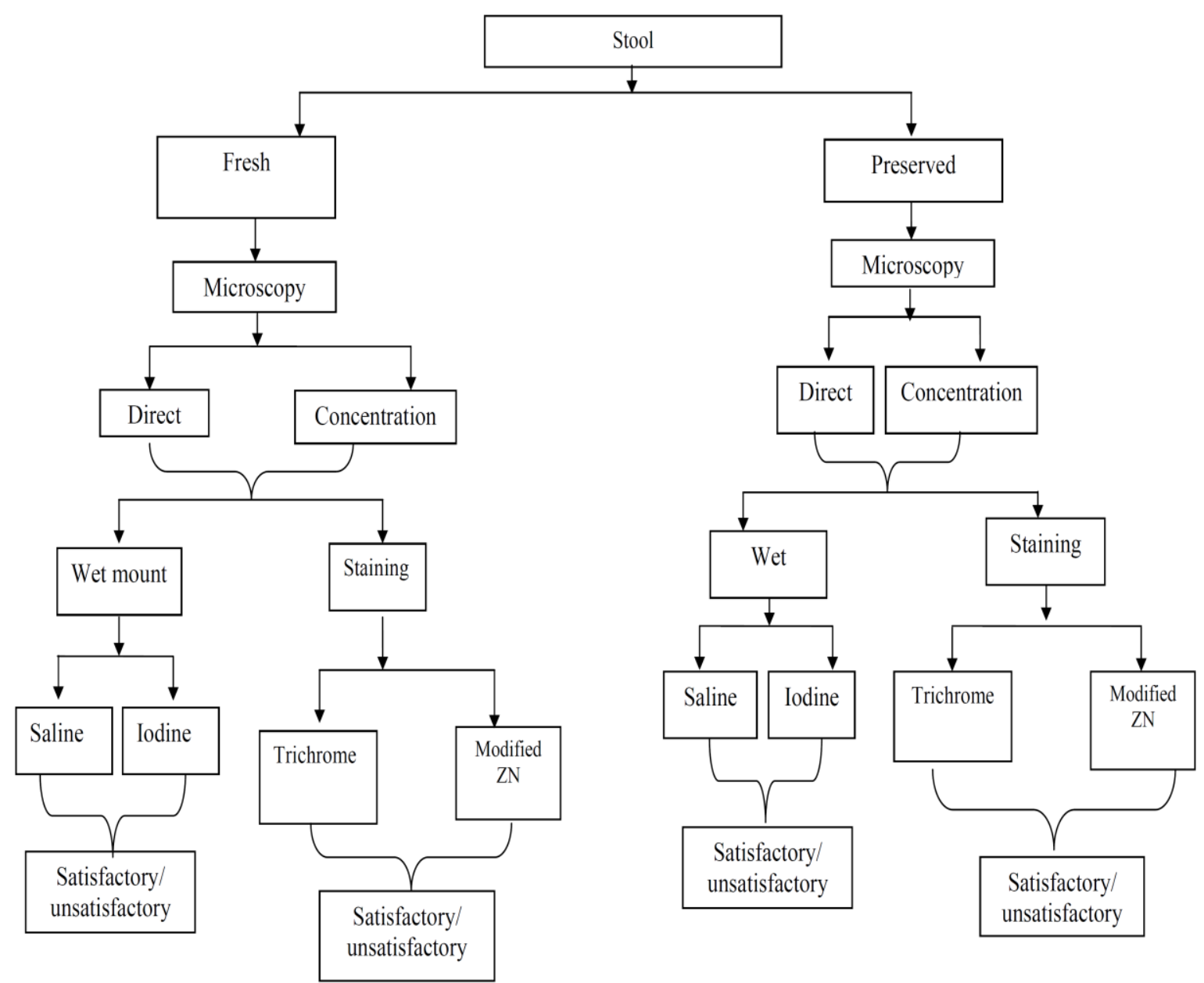


Figure.2 Cyst of Giardia lamblia in pre-preserved sample (Direct wet mount, 40x)

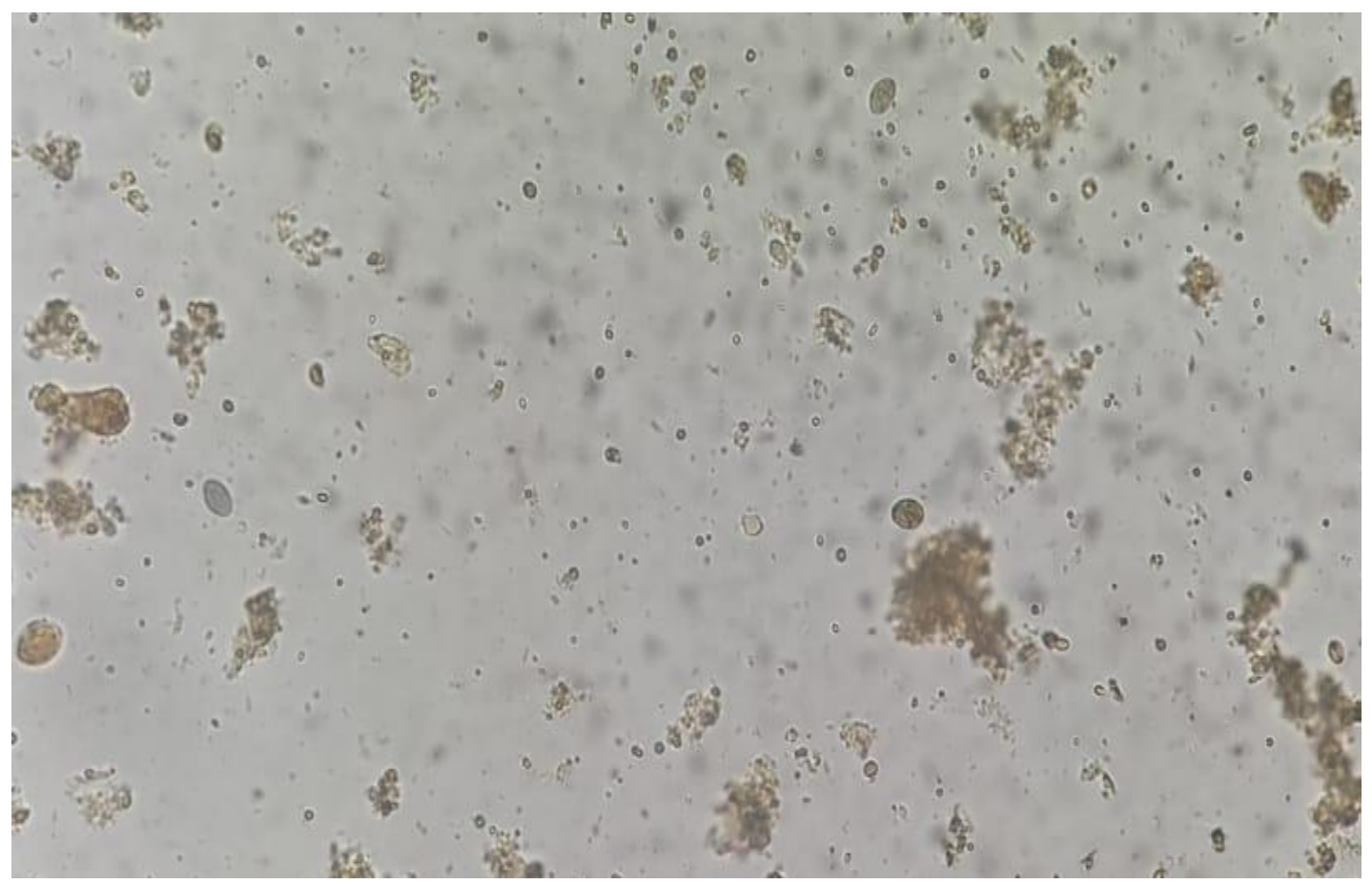

Figure.3 Egg of Hymenolepis nana in pre-preserved sample (Direct Iodine wet mount, 40x)

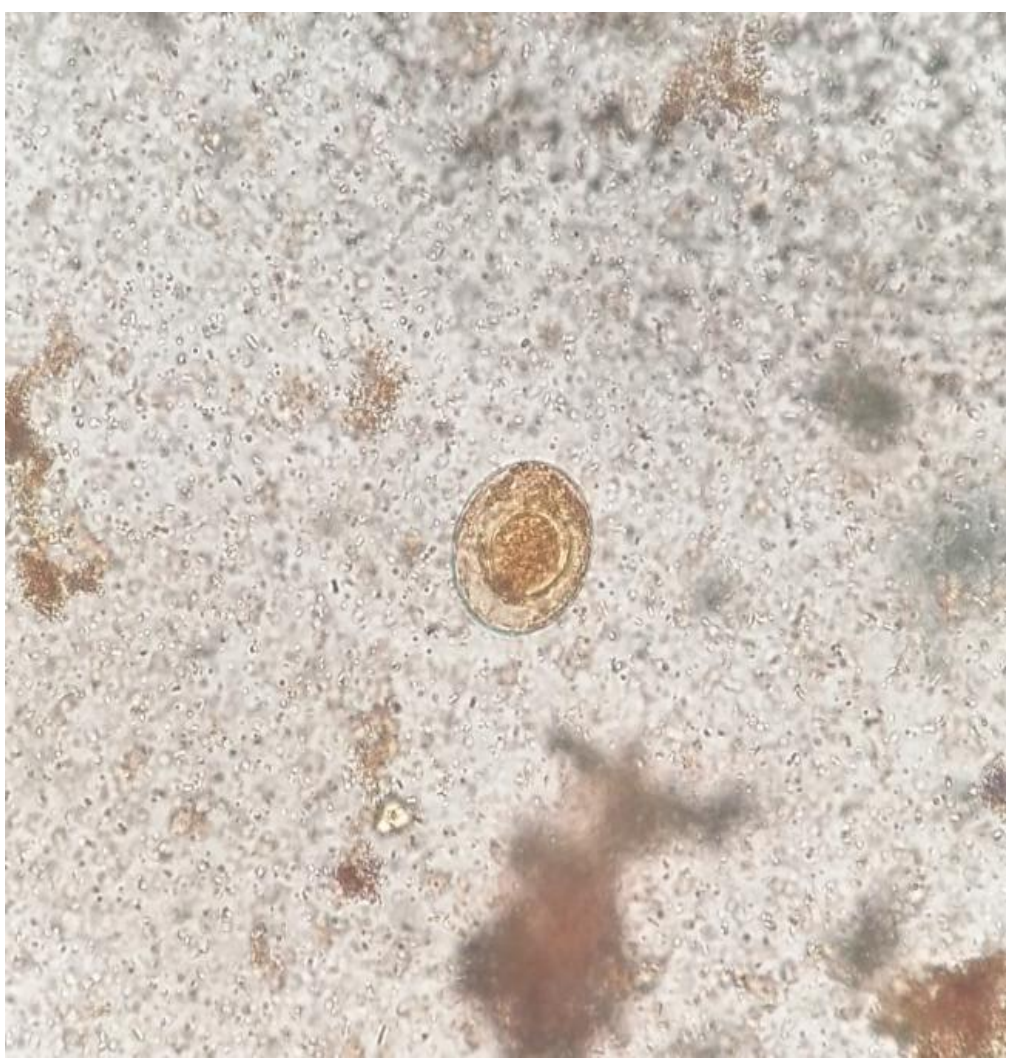


Figure.4 Egg of Hymenolepis nana in post preserved sample ((40 X) (a),(c)- Saline wet mount, (b)- Iodine wet mount, a - 10\% formalin, b - LV-PVA, c - SAF)

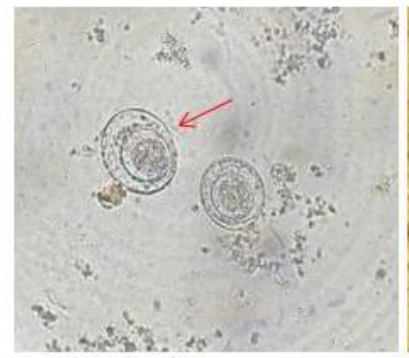

(a)

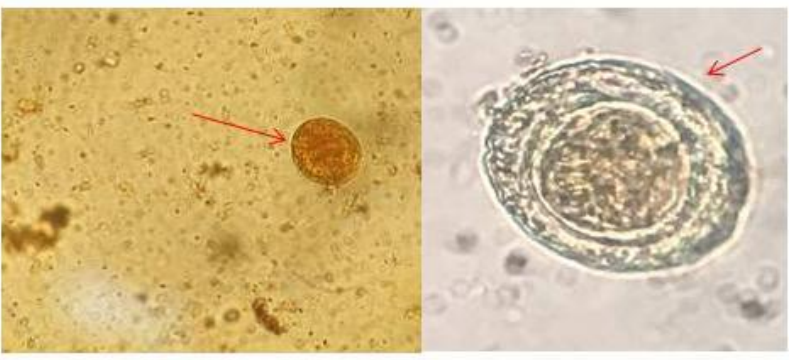

(b) (c)

Figure.5 Egg of hookworm in post preserved sample (Saline wet mount, (40 X), a - 10\% formalin, $b$ - SAF, c -LV-PVA)

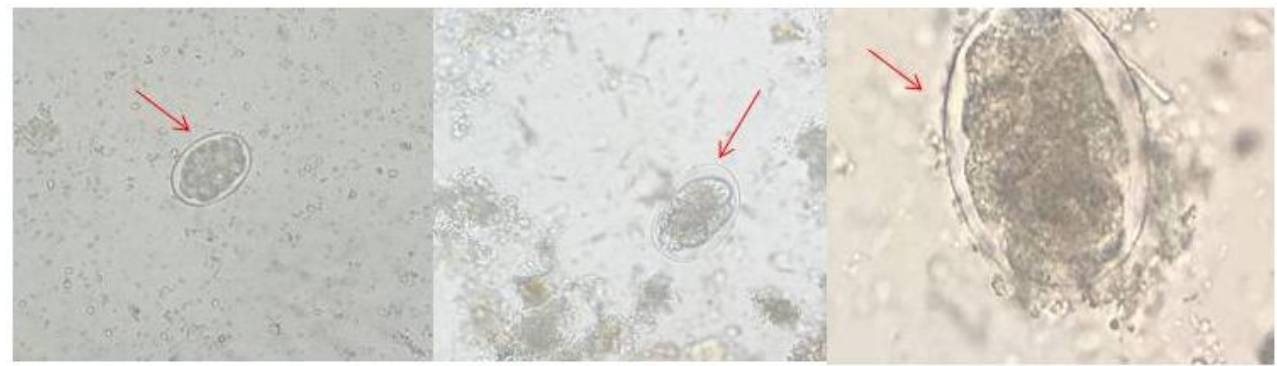

a

The presence of intestinal parasitic infections are one of the major public health problem among children (11). So, the diagnosis of parasitic infections and the preservation of parasites are very important. Different preservatives were tried for the better preservation of parasites in stool specimen. They are $10 \%$ formalin, low viscosity polyvinyl alcohol and sodium acetate acetic acid formalin, Parasafe $^{\circledR}$, Protofix $^{\circledR}$, Ecofix $^{\circledR}$ etc (11). A total number of 25 positive stool samples were used in this study. We observed that concentration method done by formol ether method gave better results in identification than that of the direct stool sample. The morphology was better for identification by iodine wet mount than saline since the internal structure was well observed b

C

in iodine wet mount. The helminth eggs were better stained by trichrome than the protozoan cyst. The distorted morphology and non-take up of stain leads to difficulty in confirmation.

Of the three preservatives used, it was observed that $10 \%$ formalin was able to preserve the morphology of parasites better than LV- PVA or SAF after a period of one month. Another study on stool preservatives also came to a conclusion that formalin and sodium acetate acetic acid was good for the preservation of stool parasites, but the health concern for laboratory personnel need to be taken care (12). It was also observed that SAF works well in concentration procedure (12). The main problem observed was the presence of parasitic forms in some of the wet mounts 
and their absence in the others. It was observed to be due to the less number of organisms (12). Similar observation was seen in our study too where the direct yielded less or minimal parasites compared to that after concentration.

A study on the comparison of fresh stool versus SAF reveals that SAF were able to diagnose more compared to fresh specimen (13). A total number of 247 stools were used for this study. The specimen was aliquoted in two containers, one for fresh specimen and the other containing SAF. On analysis by this method, only 149 of 247 were identified from SAF whereas only 89 were identified from the unpreserved samples (13). However in our study, when fresh samples can recover the parasites in all the samples, SAF could recover only 22 out of 25 . This can be due to the less density of organism per slide or maybe due to disintegration of the preserved parasite.

Another study on intestinal parasites evaluated the commercially available preservatives which are used for the detection of helminth eggs and protozoa (4). The number of samples used in the study was 20 and the stool samples had multiple stages of following parasites. They were eggs of Ascaris lumbricoides and Trichuris trichiura, larvae of Strongyloides stercoralis and cysts of Blastocystis hominis, Endolimax nana, Entamoeba coli,Entamoeba histolytica /E.dispar, Iodamoeba butschlii and Giardia intestinalis(a few trophozoites of these organisms were also found). Concentration procedure was done for all specimens which were preserved in seven different preservatives like $10 \%$ formalin, low viscosity PVA, Ecofix ${ }^{\circledR}$, Sodium acetate-acetic acid-formalin, STF, Parasafe ${ }^{\circledR}$ andprotofix ${ }^{\circledR}$. Of this, formalin, Ecofix ${ }^{\circledR}$, SAF, STF gave satisfactory results in the concentration procedures performed for wet mounts. During examination, few discrepancies were noted in the examination of various preservatives. The presence of organisms in some wet mount and the absence of the same in other wet mounts may be due to the less number of parasites per slide. This was more detrimental in case of Strongyloides stercoralis larvae and cyst of Endolimax nana or Blastocystis hominis. The color changes observed in permanent staining done from different preservatives neither aided the identification of organisms (4). It was also observed that low viscosity PVA, when stained with trichrome, were red. Such similar finding was also seen our study making this preservative not suitable for permanent staining purposes. For the other preservatives, materials preserved in SAF and stained with Iron hematoxylin were brownish -grayish (4).

Another study compared LV PVA with mercuric chloride and LV PVA with zinc sulfate was done, it observed that the best nuclear or cytoplasmic detail and clarity was seen with mercuric chloride based preservative (14). However in case of our study, it is observed that the identification of parasitic form was not possible in trichrome stain due to the distorted morphology or due to the less number of parasites per slide. There was only minimal difference in color of the smears made from both preservatives. The color obtained from mercuric chloride preserved sample was better than the same from zinc sulfate preserved sample smears. The difficult ones to identify in zinc sulfate preserved samples were the cyst forms of the parasites. The range of colors varies from pink, red, purple, blue, green. The stained smears from zinc sulfate was more green and that of mercuric chloride based were more uniformly blue with better differential colors (red, purple, pink) (14). The nuclear and cytoplasmic details of parasitic forms were different in both smears. Clear, well defined morphologic details were better in mercuric chloride based preservative. Same clarity was not always observed in case of smears 
prepared from zinc sulfate. It is observed that the number of organism also plays an important role for identification (14). Once it is observed that when organisms were rare, they were identified in mercuric chloride based preservative but not in zinc sulfate based one. It indicated that the change in morphology and the color difference along with very low load of parasitic forms prevent the identification of organism (15).

\section{Limitations}

Preservative duration of our study was only one month, because of the shortage of time and lesser quantity of stool samples. (2)We have performed only one permanent staining technique because of lack of resources. (3)Commercial preservatives were not compared with the preservatives used in our study due to the lack of time and resources.

In conclusion, use of stool preservatives help us to retain the intestinal parasites (egg/cyst) for a longer time. Of all the three preservatives used in this study, the best result was seen with $10 \%$ formalin and least with LV-PVA. The majority of problem leading to unsatisfactory result in the LV- PVA were the non-taking up of the permanent stain followed by the distortion of the morphology. So, it can be used as a short term measure for preservation though fresh sample is the best for morphology identification especially for trophozoites.

\section{References}

1. Wegayehu T, Tsalla T, Seifu B, Teklu T. Prevalence of intestinal parasitic infections among highland and lowland dwellers in Gamo area, South Ethiopia. BMC Public Health 2013;13:151

2. Hailegebriel T. Prevalence of intestinal parasitic infections and associated risk factors among students at Dona Berber primary school, Bahir Dar, Ethiopia. BMC Infect Dis 2017; 17:362

3. Khanna V, Tilak K, Rasheed S, Mukhopadhyay C. Identification and Preservation of Intestinal Parasites Using Methylene Blue-Glycerol Mount: A New Approach to Stool Microscopy. J Parasitol Res. 2014;2014:1-4.

4. Pietrzak-Johnston SM, Bishop H, Wahlquist S, Moura H. Evaluation of Commercially Available Preservatives for Laboratory Detection of Helminths and Protozoa in Human Fecal Specimens. J Clin Microbiol 2000;38:6.

5. Garcia LS. Diagnostic medical parasitology. 5th ed. Washington, D.C: ASM Press; 2007. 1202 p.

6. Garcia LS, Shimizu RY, Brewer TC, Bruckner DA. Evaluation of Intestinal Parasite Morphology in Polyvinyl Alcohol Preservative: Comparison of Copper Sulfate and Mercuric Chloride Bases for Use in Schaudinn Fixative. 1983;17:4.

7. Anamnart W, Intapan PM, Maleewong W. Modified Formalin-Ether Concentration Technique for Diagnosis of Human Strongyloidiasis. Korean J Parasitol. 2013;51:743-5.

8. Shoaib S, Hafiz A, Tauheed S. Role of Trichrome Staining Techniques in the diagnosis of Intestinal Parasitic Infections. J Pak Med Assoc 2002;52:152-4.

9. Manser MM, Saez ACS, Chiodini PL. Faecal Parasitology: Concentration Methodology Needs to be Better Standardised. PLoS Negl Trop Dis. 2016;10:e0004579.

10. Vohra P, Sharma M, Chaudhary U. A comprehensive review of diagnostic techniques for detection of Cryptosporidium parvum in stool samples. IOSR J Pharm 2012;2:15-26.

11. Ibrahim SA, Karanja S, Kombe $\mathrm{Y}$. Prevalence of intestinal parasitic 
infections and their relation with socioeconomic factors and hygienic habits among workers from high end hotels in Nairobi Kenya. J Health Med Nur 2017;13:71-83

12. Mank TG, Zaat JOM, Blotkamp J, Polderman AM. Comparison of fresh versus sodium acetate acetic acid formalin preserved stool specimens for diagnosis of intestinal protozoal infections. Eur J Clin Microbiol Infect Dis. 1995;14:1076-81.

13. Garcia LS, Shimizu RY, Shum A, Bruckner DA. Evaluation of Intestinal Protozoan Morphology in Polyvinyl Alcohol Preservative: Comparison of Zinc Sulfate- and Mercuric Chloride-
Based Compounds for Use in Schaudinn's Fixative. J Clin Microbiol 1993;31:307-10

14. Papaiakovou M, Pilotte N, Baumer B, Grant J, Asbjornsdottir K, Schaer F, et al., A comparative analysis of preservation techniques for the optimal molecular detection of hookworm DNA in a human fecal specimen. PLoS Negl Trop Dis 2018;12:e006130.

15. Jensen B, Kepley W, Guarner J, Anderson K, Anderson D, Clairmont J. 2000. Comparison of Polyvinyl Alcohol Fixative with Three Less Hazardous Fixatives for Detection and Identification of Intestinal Parasites. J Clin Microbiol.38:7.

\section{How to cite this article:}

Maneesha, P.P., Nonika Rajkumari, R. Sneha, Shashiraja Padukone and Ajay Philips Selvaratthinam. 2019. Comparison of the Use of Three Different Stool Preservatives on the Morphology of Intestinal Parasites. Int.J.Curr.Microbiol.App.Sci. 8(09): 868-878. doi: https://doi.org/10.20546/ijcmas.2019.809.104 